\title{
NLRP3 inflammasome activation mediates sleep deprivation- induced pyroptosis in mice
}

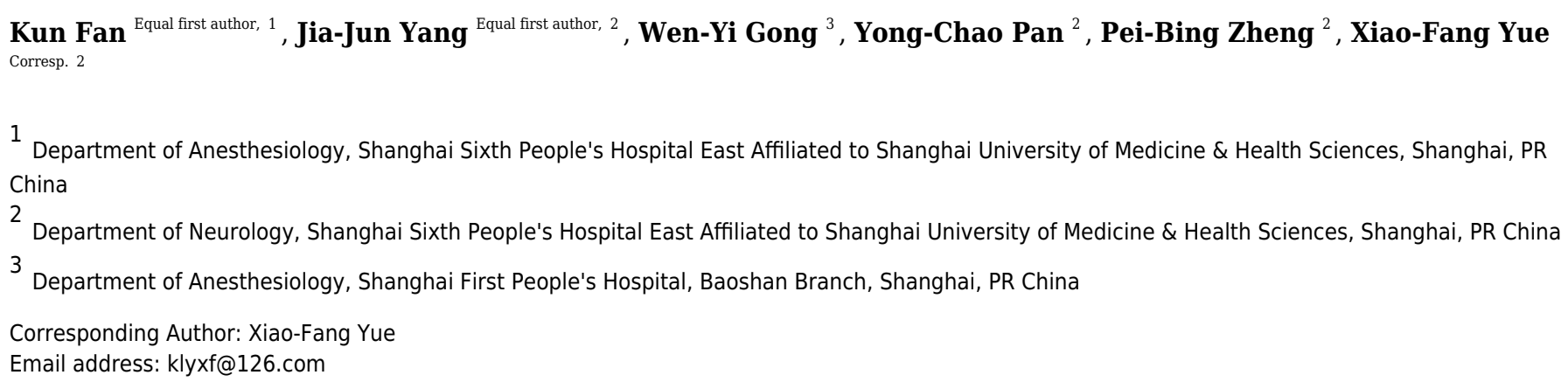

Background. Sleep deprivation (SD) has many deleterious health effects, including cognitive decline, work ability decline, inadequate alertness etc. Neuroinflammation plays an important role in sleep deprivation. However, the underlying mechanism is still unclear. Methods. In the present study, we detected the activation of microglia and apoptosis of nerve cells in sleep deprivation (SD) mice model using IHC, HE staining and TUNEL apoptosis assay. RT-PCR array data were used to detect the expression of inflammatory bodies in hippocampal CA1 region after sleep deprivation, to explore how NLRP3 inflammasome regulates neuronal apoptosis and how specific signaling pathways are involved in SD-induced activation of NLRP3/pyrosis axis. Results. We found the number of microglia significantly increased in SD mice, while this effect was blocked by sleep recovery. RT-PCR array data suggested that NLRP3 inflammasome, but not other inflammasomes, was obviously increased in hippocampus CA1 region after sleep deprivation. Mechanistically, we found that NLRP3 mediated the pyroptosis of neurocyte through GSDMD-dependent way, and P38 and ERK-MAPK signaling pathway is involved in SD induced activation of NLRP3/pyroptosis axis. All these results suggested that MAPK/NLRP3 axis mediated SD-induced pyroptosis. Conclusion. NLRP3 plays an important role in SD-induced neuroinflammation. Thus, NLRP3 inflammasome is expected to be a potential therapeutic target for SD induced neuroinflammation. 
1 NLRP3 inflammasome activation mediates sleep deprivation-induced pyroptosis in mice

2 Kun Fan ${ }^{1, \#, ~ J i a-J u n ~ Y a n g, " \#, ~ W e n-Y i ~ G o n g ³, ~ Y o n g-C h a o ~ P a n ~}{ }^{2}$, Pei-Bing Zheng ${ }^{2}$, Xiao-Fang

3 $\mathrm{Yue}^{2, *}$

\section{4 \# equal contribution}

51 Department of Anesthesiology, Shanghai Sixth People's Hospital East Affiliated to Shanghai

6 University of Medicine \& Health Sciences, Shanghai, PR China;

72 Department of Neurology, Shanghai Sixth People's Hospital East Affiliated to Shanghai

8 University of Medicine \& Health Sciences, Shanghai, PR China;

93 Department of Anesthesiology, Shanghai First People's Hospital, Baoshan Branch, Shanghai, 10 PR China.

*correspondence to: Xiao-Fang Yue, M.D. Department of Neurology, Shanghai Sixth People's 
Background. Sleep deprivation (SD) has many deleterious health effects, including cognitive decline, work ability decline, inadequate alertness etc. Neuroinflammation plays an important role in sleep deprivation. However, the underlying mechanism is still unclear.

Methods. In the present study, we detected the activation of microglia and apoptosis of nerve cells in sleep deprivation (SD) mice model using IHC, HE staining and TUNEL apoptosis assay. RTPCR array data were used to detect the expression of inflammatory bodies in hippocampal CA1 region after sleep deprivation, to explore how NLRP3 inflammasome regulates neuronal apoptosis and how specific signaling pathways are involved in SD-induced activation of NLRP3/pyrosis axis.

Results. We found the number of microglia significantly increased in SD mice, while this effect was blocked by sleep recovery. RT-PCR array data suggested that NLRP3 inflammasome, but not other inflammasomes, was obviously increased in hippocampus CA1 region after sleep deprivation. Mechanistically, we found that NLRP3 mediated the pyroptosis of neurocyte through GSDMD-dependent way, and P38 and ERK-MAPK signaling pathway is involved in SD induced activation of NLRP3/pyroptosis axis. All these results suggested that MAPK/NLRP3 axis mediated SD-induced pyroptosis.

Conclusion. NLRP3 plays an important role in SD-induced neuroinflammation. Thus, NLRP3 inflammasome is expected to be a potential therapeutic target for SD induced neuroinflammation.

Keywords: Sleep deprivation, NLRP3, Pyroptosis, MAPK. 


\section{INTRODUCTION}

38 Sleep deprivation (SD) refers to the abnormal sleep volume and abnormal behavior during sleep,

39 as well as the normal rhythmic alternation disorder of sleep and awakening. Severe SD can lead to

40 impaired neurogenesis, metabolic and cardiovascular problems, decreased immune function,

41 weakened resistance, impaired memory, and disruption of the blood-brain barrier. At present, the

42 treatment of sleep disorders is mainly divided into drug treatment and non-drug treatment.

43 However, there is a lack of research on the diagnosis and treatment of sleep disorders in patients,

44 and the underlying mechanism has not been better understood.

45 Interleukin-1 (IL-1) and tumor necrosis factor-a (TNF-a) have been extensively studied to be

46 involved in regulating physiological sleep (Hurtado-Alvarado et al. 2013), and the brain

47 communicates with the external circulatory system through lymphoid tissue or blood-brain barrier

48 (Besedovsky et al. 2012). When glial cells in the nervous system release proinflammatory factors, the proinflammatory factors induce suppression of splenic natural killer (NK) cell activity, thereby affecting the function of the nervous system (Katafuchi et al. 2009). SD has been shown to cause disorders of the immune system and activation of inflammatory responses. For example, human experiments have found that the number of white blood cells, monocytes, and neutrophils in the peripheral blood of chronic sleep deprivers is increased (Vgontzas et al. 2004). At the same time, the expression of sleep-related inflammatory factors such as IL-1 $\beta$, IL-6 and TNF-a increased to different degrees after SD (Chennaoui et al. 2014; Irwin et al. 2010). Recently, there is increasing evidence that SD causes neuroinflammation. However, the mechanism of SD-induced neuroinflammation has not been elucidated. 
domain and leucine-rich repeat protein-3 (NLRP3) inflammasome is one of the most widely

studied inflammasome receptor molecules, which consists of nucleotide-binding oligomerization domain (NACHT), apoptosis-speck-like protein (ASC) and procaspase-1 protein. Upon activation,

NLRP3 recruits the adaptor molecule ASC, which in turn recruits the cysteine protease caspase-1.

Caspase- 1 activation subsequently induces the processing of pro-IL-1 $\beta$ and pro-IL-18 into their mature secreted forms (Zhou et al. 2018). Studies have shown that NLRP3 inflammasomemediated neuroinflammatory response is closely related to the occurrence and development of neurological diseases (Shichita et al. 2014). NLRP3 inflammasome can act as a tissue damage receptor, which senses bioenergy deficiency, acidosis and oxidative stress. It regulates the maturation and secretion of IL-1beta and IL-18 by activating Procaspase- 1 to cleave into p20 and p10 subunits, and induces cell death. This type of programmed death triggered by inflammasomes is also called pyroptosis (Evavold \& Kagan 2019). A characteristic sign of pyroptosis is membrane rupture, with the release of pro-inflammatory mediators such as IL-1beta and IL-18 outside the cell. In addition, studies have shown that once the NLRP3 inflammasome is activated, the expression level of GSDMD is increased, and the cleavage of GSDMD-NT is partially dependent on the activation of caspase-1 and the Asp280 amino acid site in its structural domain, and GSDMD-NT can bind to cell membrane phospholipids through its Glu15 and Leu156 amino acid sites, then destroy cell membrane and induce pyroptosis (Rathkey et al. 2017).

In our study, we explored the effect of SD induced histological damage and the microglia by using mice model of SD. Also, we assessed the involvement of the NLRP3/caspase 1 
79

80

inflammasome pathway in SD-induced neurological injury, and further revealed the potential mechanisms underlying the NLRP3, pyroptosis and MAPK signaling pathway under SD. Taken together, all results demonstrate that NLRP3 inflammasome activation mediates SD-induced pyroptosis in SD mice.

\section{MATERIALS AND METHODS}

\section{Animal}

Adult male C57BL/6 mice (23-25 g; $\mathrm{n}=10$ of each group) were obtained from Shanghai Model Organisms Center, Inc. (Shanghai, China), and housed in plexiglass cage under standard environmental conditions of temperature $\left(25 \pm 2{ }^{\circ} \mathrm{C}\right)$ and humidity $(55 \pm 2 \% \mathrm{RH})$. The food and water were provided ad libitum and the related animal experiments were approved by the Animal Care Committee at the Shanghai First People's Hospital, Baoshan Branch (No. 2020-Y11). Animal experiments were conducted during daytime light hours.

\section{Sleep deprivation and recovery model}

SD model was performed based on our preliminary results and previous relevant reports (Franken et al. 1991; Xia et al. 2017). In brief, SD was induced for 6 hours, which began at 7 a.m. and ended at 1 p.m. Animals in the sham group were kept undisturbed in a separate room with the same light/dark cycle as the SD group. The treatment with SD was continued for 1-5 weeks. At the end of the fifth week, mice in the sleep recovery group were allowed to sleep for 24 hours. SD mice were anesthetized using $10 \%$ chloral hydrate and sacrificed by cervical dislocation, the hippocampus region was isolated from the whole brain kept on ice, washed with $0.1 \mathrm{M}$ phosphate- 
100

101

102

103

104

105

106

107

108

109

110

111

112

113

114

115

116

117

118

119

120

buffered saline solution and stored at $-80{ }^{\circ} \mathrm{C}$ or stored in $4 \%$ PFA at $4{ }^{\circ} \mathrm{C}$ for further experiments.

All mice were maintained under specific-pathogen free conditions and fed ad libitum a standard

chow. The block experiment, SD mice were treated with or without SB203580 ( $1 \mu \mathrm{mol} / \mathrm{L}$, inhibitor of p38) and U0126 (1 $\mu \mathrm{mol} / \mathrm{L}$, inhibitor of Erk).

\section{Hematoxylin and Eosin (HE) Staining}

Hippocampus CA1 region was immersed in 4\% paraformaldehyde and then embedded in paraffin.

The tissue was cut into 4-mm sections and HE staining was carried out as follows: The section was stained with hematoxylin for $5 \mathrm{~min}$, decolorized with $75 \%$ hydrochloric acid and alcohol solution for $30 \mathrm{~s}$, stained with eosin for $5 \mathrm{~min}$, and finally decolorized with $90 \%$ ethanol for $35 \mathrm{~s}$.

\section{Immunohistochemistry (IHC)}

Paraffin sections were then dewaxed and hydrated and then washed with PBS at room temperature.

Heat mediated antigen retrieval with Tris/EDTA buffer $\mathrm{pH} 9.0$ was performed. Then, the sections were washed with PBS three times and treated with $3 \% \mathrm{H}_{2} \mathrm{O}_{2}$-methanol for 15 min. Immunostaining was carried out through incubation with antibodies against IBA-1 (4 $\mu \mathrm{g} / \mathrm{ml}$, ab48004, Abcam), NLRP3 (1:500, ab214185, Abcam) and GSDMD (1:1000, ab219800, Abcam).

\section{Immunofluorescence}

The brain cryosections were first incubated with $70 \%$ Ethanol for $5 \mathrm{~min}$ at room temperature, then washed with PBS and incubated for $40 \mathrm{~s}$ with $40 \mathrm{mg} / \mathrm{ml}$ Proteinase K, and blocked for 1 hour at room temperature in blocking buffer $(0.3 \%$ Triton X-100/10\% Goat serum/phosphate buffer saline), and incubated with primary antibodies overnight at $4{ }^{\circ} \mathrm{C}$ in blocking buffer. Sections were then washed 3 times in blocking buffer and secondary antibodies were incubated for 1 hour at 
121

122

123

124

125

126

127

128

129

130

131

132

133

134

135

136

137

138

139

140

141

room temperature in the dark. Sections were washed again, incubated with DAPI for $5 \mathrm{~min}$ at room temperature, washed and mounted in Citifluor (Agar Scientific).

Primary antibodies used for Immunofluorescence were as follow: NeuN (1:1000, ABN78, sigma) All secondary antibodies were also purchased from Abcam.

\section{TdT-mediated dUTP-biotin nick end labeling (TUNEL) staining}

TUNEL staining was carried out using the In Situ Cell Death Detection Kit (Sigma, St. Louis, MO, USA). The sections were fixed in $4 \%$ paraformaldehyde for $1 \mathrm{~h}$ and treated with proteinase $\mathrm{K}$ (20 $\mu \mathrm{g} / \mathrm{ml}$ ) for $15 \mathrm{~min}$ at $37^{\circ} \mathrm{C}$. Peroxidase blocking with $0.3 \%$ hydrogen peroxide in methanol, permeation with $0.1 \%$ sodium citrate in $0.1 \%$ Triton $\mathrm{X}-100$ for 2 minutes. Then, the sections were then incubated with TUNEL solution from In Situ Cell Death Detection Kit for $1 \mathrm{~h}$ at $37^{\circ} \mathrm{C}$. The sections were examined using a Nikon Eclipse E6000 fluorescent microscope.

\section{ELISA assay}

Hippocampus CA1 region tissue samples were gathered for the detection of IL-1 $\beta$, IL-18 and TNF- $\alpha$ concentrations by ELISA kit (Solarbio). All the tests were carried out as the manufacturer's instructions. Each sample was tested in triplicate.

\section{Western Blot analysis}

Hippocampus CA1 region was lysed with RIPA Buffer (Solarbio, Beijing, China), and the total protein concentration was quantified with BCA protein assay kit (Pierce, Rockford, IL, USA). 30 $\mu \mathrm{g}$ of total extract proteins were separated on $10 \%$ SDS-PAGE and transferred to PVDF membranes (Roche, Basel, Switzerland). The membranes were incubated with primary antibodies against NLRP3 (1:1000, ab214185, Abcam), Caspase-1 (1 $\mu \mathrm{g} / \mathrm{ml}$, ab138483, Abcam), GSDMD 
142 (1:1000, ab219800, Abcam), ASC (1:1000, ab167165, Abcam), p-P38 (1:1000, ab178867,

143 Abcam), P38 (1:1000, ab170099, Abcam), p-ERK (1:1000, ab201015, Abcam), ERK (1:1000,

144 ab32537, Abcam), p-AKT (1:1000, ab38449, Abcam), AKT (1:500, ab8805, Abcam) and $\beta$-actin

145 (1:5000, ab6276, Abcam) overnight at $4{ }^{\circ} \mathrm{C}$. The membranes were incubated with HRP-conjugated

146 secondary anti-rabbit (1:5000) for $1 \mathrm{~h}$ at room temperature and then visualized by ECL kit

147 (Solarbio). The intensity of bands was determined using software ImageJ (NIH, Bethesda, MA, 148 USA).

149 Quantitative reverse transcription (qRT-PCR)

150 Total RNA was extracted from hippocampus CA1 region of the control group, SD group and SD

151 recovery group by Trizol reagent (Solarbio) as the manufacturer's instructions. Reverse 152 transcriptional PCR was carried out with the iScripe ${ }^{\mathrm{TM}}$ cDNA Synthesis kit (Bio-Rad, Hercules, 153 CA, USA). qPCR reaction was performed using an ABI 7500-Fast Real-Time PCR System 154 (Applied Biosystem, Foster City, CA, USA) using LightCycler 480 SYBR Mix (Roche). The fold 155 changes were calculated with the $2^{-\Delta \Delta \mathrm{Ct}}$ method and GAPDH served as a normalizing control.

156 Statistical analysis

157 All data are expressed as mean \pm SD of three independent experiments. Statistical analyses were 158 performed with the SPSS 19.0 statistical software (IBM, Armonk, NY, USA). Data was assessed 159 for normality using D'Agostino and Pearson omnibus normality test. Values among multiple 160 groups were compared via one-way ANOVA, and post hoc comparisons were conducted by the 161 Bonferroni test or by Dunnett method if the homogeneity of variance was not met. $\mathrm{P}<0.05$ was 162 considered statistically significant. 
163

164

165

166

167

168

169

170

171

172

173

174

175

176

177

178

179

180

181

182

183

\section{RESULTS}

\section{Sleep deprivation induced the increase of microglia and neuronal damage}

To evaluate the effect of sleep deprivation induced histological damage, we did HE, Tunel staining and IHC staining for IBA-1, the marker of microglia, on brain sections of the hippocampus CA1 region. As shown in Figure 1A and B, the average number of microglia was obviously increased in SD group compared with control group, while the average number of microglia decreased after 24h sleep recovery. H\&E staining data showed that the nuclear pyknosis was significantly increased in SD group compared with control group, while the effect was blocked by sleep recovery (Figure 1C and D). Consistently, the Tunel staining data showed that the number of apoptosis cells in SD group is significantly higher than that in control group, and restored by sleep recovery (Figure 1E and F). These data suggest that SD may mediate neuronal cell death through microglia activation.

\section{Sleep deprivation promoted the activation of NLRP3/caspase 1 pathway.}

The microglia play an important role in neuroinflammation, and the inflammasome, which leads to caspase- 1 activation, is implicated in neuroinflammation. To determine which inflammasome pathway is involved in sleep deprivation induced neuronal damage, inflammasome RT-PCR array was performed. As shown in Figure 2A, only NLRP3 mRNA levels were significantly increased in SD group, while NLRP1, NLRP2 and AIM2 were not significantly increased in SD group. To further confirmed the data, we measured the protein level of NLRP3, ASC and caspase-1, markers of the NLRP3 inflammasome pathway, in brain tissue. Our results showed that the protein levels of NLRP3, ASC and activated caspase-1 were higher at SD group compared to control group, and 
184 185 186 187

decreased by sleep recovery (Figure 2B and C). Consistently, the IHC staining for NLRP3 also showed the NLRP3 obviously increased in SD group compared with control group, and restored by sleep recovery (Figure 2D).

\section{Sleep deprivation induced the pyroptosis of neuronal cell}

Activated caspase-1 indicates the presence of pyroptosis. Caspase-1 regulates the cleavage and maturation of the downstream inflammatory cytokines IL-1 $\beta$ and IL-18. To evaluate the effect of Sleep deprivation on hippocampal inflammation response, The mRNA and protein level of IL-1 $\beta$, IL-18, TNF- $\alpha$ was further confirmed in hippocampus CA1 region by RT-PCR and Elisa. As shown in Figure 3A and B, the level of IL-1 $\beta$, IL-18, TNF- $\alpha$ was markedly increased in SD group, while the effect was blocked by sleep recovery. Besides, the protein level of pyroptosis marker GSDMD and apoptosis marker bax were analyzed by Western-blotting, the data revealed a significant increase in the expression of GSDMD and bax in the mouse brain of hippocampus CA1 region, and the expression of GSDMD, but not the expression of bax, was reversed by $24 \mathrm{~h}$ sleep recovery (Figure 3C-D). We further detected the GSDMD expression by Immunohistochemistry. As shown in Figure 3E, the expression of GSDMD was markedly increased in SD group, while the effect was blocked by sleep recovery. To further confirmed the pyroptosis cell type, the mouse brain of hippocampus CA1 region was stained with the neuronal marker NeuN and GSDMD. As shown in the Figure 3F, brains showed colocalization of the neuronal marker NeuN with GSDMD. These data suggested that SD induced the pyroptosis of neuronal cell.

\section{Sleep deprivation induces the activation of P38 and ERK MAPKs pathway}

It is well-known that the MAPKs and AKT activation is related to the of NLRP3 and cell 
205

206

207

208

209

210

211

212

213

214

215

216

217

218

219

220

221

222

223

224

225

pyroptosis (Zhang et al. 2020; Zhou et al. 2019). Thus, we detected the activation of MAPKs

pathways in control and SD model with or without $24 \mathrm{~h}$ sleep recovery brain tissue of hippocampus

CA1 region. Intriguingly, we found that phosphorylation of $\mathrm{p} 38$ and ERK1/2 was increased after sleep deprivation but not AKT phosphorylation compared with the control group, while sleep recovery significantly inhibited p38 and ERK1/2 phosphorylation (Figure 4A-F). To further confirmed the p38 and ERK1/2 pathway in the SD induced NLRP3 and pyroptosis activation, the SD mice were treated with or without the p38 and ERK pathway inhibitor SB203580 and U0126, and the NLRP3 and pyroptosis activation was analyzed by Western blot. As shown in Figure 4 G and $\mathrm{H}$, the p38 inhibitor SB203580 and the ERK inhibitor U0126 could significantly block the SD induced activation of NLRP3 and neuronal pyroptosis.

\section{DISCUSSION}

Sleep deprivation (SD) is very common, with $20 \%$ of adults reported to be sleep deprived (Bandyopadhyay \& Sigua 2019). SD has many harmful effects, including increased risk of stroke, adiposity, glycuresis, tumor, permanent cognitive impairment, osteoporosis, cardiovascular disease and mortality (Tobaldini et al. 2017). Increasing evidence suggest that SD causes neuroinflammation in the brain (Xue et al. 2019). However, the specific mechanism by which SD triggers neuroinflammation in the brain is unclear. In our study, we found that 1) SD induced the increase of microglia and neuronal damage; 2) SD promoted the activation of NLRP3/caspase 1

pathway; 3) SD induced the pyroptosis of neuronal cell; 4) SD induces the activation of P38 and ERK MAPKs pathway. The current data verify the NLRP3 inflammasome activation mediates SD-induced pyroptosis in mice. 
227 Microglia, as a kind of innate immune cells in the central nervous system, are the key links in the process of inflammation (Su et al. 2016). Microglia can be activated either directly by some toxic substances or endogenous proteins or indirectly by dying neurons. Long-term activation of microglia can lead to neuronal damage (Fakhoury 2015; Suzumura 2014). Activated microglia, on the one hand, exert the role of phagocytes in the brain through direct contact with neurons; on the other hand, secrete some inflammatory mediators such as NO, TNF-a, IL-1 and chemokines. Overactivation of microglia enlarges the inflammatory response continuously and produces a large number of cytokines and reactive oxygen species products, which further aggravates neuronal injury. In our study, we constructed SD mice model to detect the expression of IBA-1 (the marker of microglia) in the brain part of hippocampal CA1 region of SD mice by IHC. The results showed that the expression level of IBA-1 was significantly up-regulated in the hippocampal CA1 region after SD and deceased after $24 \mathrm{~h}$ sleep recovery, suggesting that SD promoted the activation of microglia. Then, the level of IL-1 $\beta$, IL-18, TNF- $\alpha$ (inflammatory factor) was further detected in hippocampus CA1 region of SD mice. The results showed that the expression of IL-1 $\beta$, IL-18, TNF- $\alpha$ was significantly increased after SD and deceased after $24 \mathrm{~h}$ sleep recovery, suggesting that SD triggers inflammation by activating microglia, leading to neuronal damage. and is characterized by Caspase- 1 activation, cell membrane pore formation, and release of cellular contents (Fang et al. 2020). Although a certain degree of pyroptosis can endow the host with a 
$247 \&$ Lamkanfi 2016). NLRs act as an intracellular pattern receptor that can activate Caspase-1 and

248 NF-kB, MAPK signaling pathways, promote the production of proinflammatory factors, and thus

249 initiate innate and acquired immunity after recognizing the corresponding ligands (Liu et al. 2019).

250 NLRP3 is an important member of the NLR family, activated caspase-1 leads to differences in the

251 formation of plasma membrane pores within and outside cells (Fink \& Cookson 2006). High

252 intracellular osmotic pressure leads to tissue fluid entering cells, resulting in swelling and

253 dissolution of cells and release of inflammatory cytokines (IL-1 $\beta$, IL-18, and TNF- $\alpha$ ) (Miggin et

254 al. 2007). Mark R Zielinski et al found that the activation of the NLRP3 inflammasome can

255 modulate sleep induced by both increased wakefulness and a bacterial component in the

256 brain(Zielinski et al. 2017). Here, we examined several inflammasome-related genes by RT-PCR

257 array to determine which inflammasome pathways are involved in neuronal damage induced by

258 SD, and the results showed that only NLRP3 mRNA levels were significantly increased in SD

259 group and the protein levels of the markers of the NLRP3 inflammasome pathway (NLRP3, ASC

260 and caspase-1) were increased, while decreased by sleep recovery. Additionally, the level of IL-

$2611 \beta$, IL-18, TNF- $\alpha$ was also significantly increased in SD group. This is consistent with the findings

262 of Xia et al. (Xia et al. 2017), who found that in SD group, SD stimulated formation of NLRP3

263 inflammasomes, with subsequent induction of the maturation of IL-1 $\beta$ and IL-18.

264 GSDMD is a member of the gasdermins (GSDMs) family, a common substrate of caspase-1

265 and caspase-4/5/11, and an executor of pyroptosis. GSDMD consists of two conserved domains:

266 The C-terminal inhibitory domain $(21 \mathrm{ku})$ and the N-terminal effector domain $(32 \mathrm{ku})$, where the

267 N-terminal is cytotoxic and can bind to lipid components to form holes in the cell membrane.

Peer] reviewing PDF | (2020:11:55599:2:0:NEW 17 May 2021) 
268 GSDMD can be cleaved into C-terminal and N-terminal by caspase-1/4/5/11, and its N-terminal

269 oligomerizes on the cell membrane to form non-selective pore channels, releasing mature IL-18

270 and IL-1 $\beta$, and inducing the occurrence of pyroptosis (He et al. 2015; Zhaolin et al. 2019). In our

271 study, the expression of GSDMD was detected in hippocampus CA1 region of SD group and 24h

272 sleep recovery group by Western Blot and IHC assay, the data revealed a significant increase in

273 the expression of GSDMD in the mouse hippocampus CA1 region of SD group, and this effect

274 was reversed by $24 \mathrm{~h}$ sleep recovery. In addition, the proptosis marker GSDMD colocalization with

275 the neuronal marker NeuN, suggesting that NLRP3 mediates the pyroptosis of neurocyte through

276 GSDMD-dependent way. To further investigate the mechanism of pyroptosis, we attempted to

277 identify the signaling pathways that regulate pyroptosis. LI et al., confirmed that blockage of p38

MAPK signaling pathway with SB203580 suppressed macrophage pyroptosis and LPS-induced

acute lung injury through negative regulation of NLRP3 inflammasome activation. In addition,

DHA ameliorated I/R-induced injury by inhibiting pyroptosis of hepatocytes induced in liver I/R injury in vivo and in vitro through the PI3K/Akt pathway. In our study, we detected the activation

of MAPKs pathways in control and SD model with or without $24 \mathrm{~h}$ sleep recovery brain tissue of hippocampus CA1 region, phosphorylation of p38 and ERK1/2 was increased after sleep deprivation but not AKT phosphorylation compared with control group, while sleep recovery significantly inhibited p38 and ERK1/2 phosphorylation. All these results suggest that P38 and ERK-MAPK signaling pathway is involved in SD induced activation of NLRP3/pyroptosis axis. and ERK-MAPK signaling pathway is involved in SD-induced activation of NLRP3/pyrosis axis 
289

290

291

292

293

294

295

296

297

298

299

300

301

302

303

304

305

306

307

308

309

remains unclear and needs further exploration. In addition, we found that most of the pyroptosis occurred in the hippocampus, and next we are going to explore the effects of SD on cognitive impairment in animals.

\section{CONCLUSIONS}

The results of our study demonstrate that MAPK/NLRP3 axis may play a vital role in neuronal pyroptosis in the development of SD. These results provide a new direction for the clinical treatment of SD.

\section{ETHICS APPROVAL AND CONSENT TO PARTICIPATE}

All experiments in this study were approved by the Animal Care Committee at the Shanghai First People's Hospital, Baoshan Branch (No. 2020-Y-11).

\section{FUNDING}

This work was supported by the National Natural Science Foundation of China [grant number 31400953]. The sponsor provided financial support for conducting experiments, analyzing data, preparing figures, and revising the manuscript.

\section{DATA AVAILABILITY STATEMENT}

The datasets used and analysed during the current study are already included in the manuscript.

\section{AUTHOR CONTRIBUTIONS}

All authors conceived and designed the experiments, performed the experiments, analyzed the data, prepared the figures and/or tables and drafted the work or revised it critically for important content. 
311 The authors declare that they have no competing interests.

\section{PATIENT CONSENT FOR PUBLICATION}

313 Not applicable.

\section{REFERENCES}

Bandyopadhyay A, and Sigua NL. 2019. What Is Sleep Deprivation? Am J Respir Crit Care Med 199:P11-P12. 10.1164/rccm.1996P11

Besedovsky L, Lange T, and Born J. 2012. Sleep and immune function. Pflugers Arch 463:121-137. 10.1007/s00424011-1044-0

Chennaoui M, Drogou C, Sauvet F, Gomez-Merino D, Scofield DE, and Nindl BC. 2014. Effect of acute sleep deprivation and recovery on Insulin-like Growth Factor-I responses and inflammatory gene expression in healthy men. Eur Cytokine Netw 25:52-57. 10.1684/ecn.2014.0356

Evavold CL, and Kagan JC. 2019. Defying Death: The (W)hole Truth about the Fate of GSDMD Pores. Immunity 50:1517. 10.1016/j.immuni.2018.12.032

Fakhoury M. 2015. Role of Immunity and Inflammation in the Pathophysiology of Neurodegenerative Diseases. Neurodegener Dis 15:63-69. 10.1159/000369933

Fang Y, Tian S, Pan Y, Li W, Wang Q, Tang Y, Yu T, Wu X, Shi Y, Ma P, and Shu Y. 2020. Pyroptosis: A new frontier in cancer. Biomed Pharmacother 121:109595. 10.1016/j.biopha.2019.109595

Fink SL, and Cookson BT. 2006. Caspase-1-dependent pore formation during pyroptosis leads to osmotic lysis of infected host macrophages. Cell Microbiol 8:1812-1825. 10.1111/j.1462-5822.2006.00751.x

Franken P, Dijk DJ, Tobler I, and Borbely AA. 1991. Sleep deprivation in rats: effects on EEG power spectra, vigilance states, and cortical temperature. Am J Physiol 261:R198-208. 10.1152/ajpregu.1991.261.1.R198

He WT, Wan H, Hu L, Chen P, Wang X, Huang Z, Yang ZH, Zhong CQ, and Han J. 2015. Gasdermin D is an executor of pyroptosis and required for interleukin-1beta secretion. Cell Res 25:1285-1298. 10.1038/cr.2015.139

Hurtado-Alvarado G, Pavon L, Castillo-Garcia SA, Hernandez ME, Dominguez-Salazar E, Velazquez-Moctezuma J, and Gomez-Gonzalez B. 2013. Sleep loss as a factor to induce cellular and molecular inflammatory variations. Clin Dev Immunol 2013:801341. 10.1155/2013/801341

Irwin MR, Carrillo C, and Olmstead R. 2010. Sleep loss activates cellular markers of inflammation: sex differences. Brain Behav Immun 24:54-57. 10.1016/j.bbi.2009.06.001

Katafuchi T, Duan S, Take S, and Yoshimura M. 2009. Cytokine-induced suppression of medial preoptic neurons: mechanisms and neuroimmunomodulatory effects. Ann N Y Acad Sci 1153:76-81. 10.1111/j.17496632.2008.03963.x 
Liu P, Lu Z, Liu L, Li R, Liang Z, Shen M, Xu H, Ren D, Ji M, Yuan S, Shang D, Zhang Y, Liu H, and Tu Z. 2019. NOD-like receptor signaling in inflammation-associated cancers: From functions to targeted therapies. Phytomedicine 64:152925. 10.1016/j.phymed.2019.152925

Miggin SM, Palsson-McDermott E, Dunne A, Jefferies C, Pinteaux E, Banahan K, Murphy C, Moynagh P, Yamamoto M, Akira S, Rothwell N, Golenbock D, Fitzgerald KA, and O'Neill LA. 2007. NF-kappaB activation by the TollIL-1 receptor domain protein MyD88 adapter-like is regulated by caspase-1. Proc Natl Acad Sci U S A 104:3372-3377. 10.1073/pnas.0608100104

Rathkey JK, Benson BL, Chirieleison SM, Yang J, Xiao TS, Dubyak GR, Huang AY, and Abbott DW. 2017. Live-cell visualization of gasdermin D-driven pyroptotic cell death. I Biol Chem 292:14649-14658. 10.1074/jbc.M117.797217

Shichita T, Ito M, and Yoshimura A. 2014. Post-ischemic inflammation regulates neural damage and protection. Front Cell Neurosci 8:319. 10.3389/fncel.2014.00319

Su P, Zhang J, Wang D, Zhao F, Cao Z, Aschner M, and Luo W. 2016. The role of autophagy in modulation of neuroinflammation in microglia. Neuroscience 319:155-167. 10.1016/j.neuroscience.2016.01.035

Suzumura A. 2014. [Microglia in neurodegenerative disorders and neuroinflammation]. Rinsho Shinkeigaku 54:11191121. 10.5692/clinicalneurol.54.1119

Tobaldini E, Costantino G, Solbiati M, Cogliati C, Kara T, Nobili L, and Montano N. 2017. Sleep, sleep deprivation, autonomic nervous system and cardiovascular diseases. Neurosci Biobehav Rev 74:321-329. 10.1016/j.neubiorev.2016.07.004

Vande Walle L, and Lamkanfi M. 2016. Pyroptosis. Curr Biol 26:R568-R572. 10.1016/j.cub.2016.02.019

Vgontzas AN, Zoumakis E, Bixler EO, Lin HM, Follett H, Kales A, and Chrousos GP. 2004. Adverse effects of modest sleep restriction on sleepiness, performance, and inflammatory cytokines. J Clin Endocrinol Metab 89:21192126. 10.1210/jc.2003-031562

Wadhwa M, Prabhakar A, Anand JP, Ray K, Prasad D, Kumar B, and Panjwani U. 2019. Complement activation sustains neuroinflammation and deteriorates adult neurogenesis and spatial memory impairment in rat hippocampus following sleep deprivation. Brain Behav Immun 82:129-144. 10.1016/j.bbi.2019.08.004

Xia M, Li X, Yang L, Ren J, Sun G, Qi S, Verkhratsky A, and Li B. 2017. The ameliorative effect of fluoxetine on neuroinflammation induced by sleep deprivation. J Neurochem. 10.1111/jnc.14272

Xue R, Wan Y, Sun X, Zhang X, Gao W, and Wu W. 2019. Nicotinic Mitigation of Neuroinflammation and Oxidative Stress After Chronic Sleep Deprivation. Front Immunol 10:2546. 10.3389/fimmu.2019.02546

Zhang FL, Zhou BW, Yan ZZ, Zhao J, Zhao BC, Liu WF, Li C, and Liu KX. 2020. 6-Gingerol attenuates macrophages pyroptosis via the inhibition of MAPK signaling pathways and predicts a good prognosis in sepsis. Cytokine 125:154854. 10.1016/j.cyto.2019.154854

Zhaolin Z, Guohua L, Shiyuan W, and Zuo W. 2019. Role of pyroptosis in cardiovascular disease. Cell Prolif 52:e12563. 10.1111/cpr.12563

Zhou R, Yang X, Li X, Qu Y, Huang Q, Sun X, and Mu D. 2019. Recombinant CC16 inhibits NLRP3/caspase-1-induced pyroptosis through p38 MAPK and ERK signaling pathways in the brain of a neonatal rat model with sepsis. J Neuroinflammation 16:239. 10.1186/s12974-019-1651-9

Zhou W, Chen C, Chen Z, Liu L, Jiang J, Wu Z, Zhao M, and Chen Y. 2018. NLRP3: A Novel Mediator in Cardiovascular Disease. J Immunol Res 2018:5702103. 10.1155/2018/5702103

Zielinski MR, Gerashchenko D, Karpova SA, Konanki V, McCarley RW, Sutterwala FS, Strecker RE, and Basheer R.

Peer) reviewing PDF | (2020:11:55599:2:0:NEW 17 May 2021) 
2017. The NLRP3 inflammasome modulates sleep and NREM sleep delta power induced by spontaneous wakefulness, sleep deprivation and lipopolysaccharide. Brain Behav Immun 62:137-150. 10.1016/j.bbi.2017.01.012

\section{FIGURE LEGENDS}

\section{Figure 1. Sleep deprivation induced the increase of microglia and neuronal damage}

(A and B) IHC for IBA-1 level in hippocampus CA1 region of control group ( $n=10)$, SD group $(n=10)$ and $S D+$ recover group $(n=10)$. Scale bars $=50 \mu \mathrm{m}$ for IHC. (C and D) H\&E staining for hippocampus CA1 region in control group $(n=10)$, SD group $(n=10)$ and SD+ recover group $(n=10)$. Scale bars $=100 \mu \mathrm{m}$ for H\&E. (E and F) Cell apoptosis were detected in hippocampus CA1 region in control group $(n=10), S D$ group $(n=10)$ and $S D+$ recover group $(n=10)$ by Tunel staining. Scale bars $=25 \mu \mathrm{m}$ for Tunel staining. Results represent the mean of three independent experiments ${ }^{*} p<0.05$. IHC, immunohistochemical; IBA-1, Ionized calcium binding adaptor molecule-1; SD, sleep deprivation; H\&E staining, hematoxylin-eosin staining.

\section{Figure 2. Sleep deprivation promoted the activation of NLRP3/caspase 1 pathway}

(A) RT-PCR array for the expression levels of genes (IL-1 $\beta$, IL-18, Caspase-1, NLRP3, Caspase4, ASC, GSDMD, NLRP1, NLRP2 and AIM2) in control group, SD group and SD+ recover group $(\mathrm{n}=5)$. (B and C) Western blot analysis for Caspase-1, ASC and NLRP3 protein level in hippocampus CA1 region of control group, SD group and SD+ recover group $(n=5)$. (D) IHC for NLRP3 level in hippocampus CA1 region of control group, SD group and SD+ recover group. Scale bars $=50 \mu \mathrm{m}$ for IHC $(n=5)$. Results represent the mean of three independent experiments ${ }^{*} p<0.05,{ }^{*} p<0.01$. RT-PCR, Quantitative real-time PCR; IHC, immunohistochemical; SD, sleep 
407 deprivation; ASC, apoptosis-associated specklike protein containing a CARD.

408 Figure 3. Sleep deprivation induced the pyroptosis of neuronal cell

409 (A and B) qPCR (A) and ELISA (B) analysis of IL-1 $\beta$, IL-18 and TNF- $\alpha$ expression in

410 hippocampus CA1 region of control group, SD group and SD+ recover group and qPCR analysis

411 of IL-1 $\beta$, IL-18 and TNF- $\alpha$ expression in hippocampus of control group, SD group and SD+

412 recover group $(\mathrm{n}=5)$. (C and D) Western blot analysis for GSDMD and bax protein level in mouse

413 hippocampus CA1 region of control group, SD group and SD+ recover group (n=5). (E) IHC for

414 GSDMD level in mouse hippocampus CA1 region of control group, SD group and SD+ recover

415 group $(n=5)$. Scale bars $=50 \mu \mathrm{m}$ for IHC. (F) Fluorescence co-localization for GSDMD and NeuN

416 in mouse hippocampus CA1 region of control group, SD group and SD+ recover group $(n=5)$.

417 Scale bars $=20 \mu \mathrm{m}$. Results represent the mean of three independent experiments $*^{*}<0.05$,

$418 * * p<0.01$. qPCR, Quantitative real-time PCR; IHC, immunohistochemical; SD, sleep deprivation.

419 Figure 4. Sleep deprivation induces the activation of P38 and ERK MAPKs pathway

420 (A and B) Western blot analysis for p-P38 and P38 protein level in mouse hippocampus CA1

421 region of control group, $\mathrm{SD}$ group and $\mathrm{SD}+$ recover group $(\mathrm{n}=5)$. (C and $\mathrm{D})$ Western blot analysis

422 for p-ERK and ERK protein level in mouse hippocampus CA1 region of control group, SD group

423 and $\mathrm{SD}+$ recover group $(\mathrm{n}=5)$. $(\mathrm{E}$ and $\mathrm{F})$ Western blot analysis for $\mathrm{p}-\mathrm{AKT}$ and AKT protein level

424 in mouse hippocampus CA1 region of control group, SD group and SD+ recover group $(n=5) .(G$

425 and $\mathrm{H}$ ) Western blot analysis for NLRP3 and GSDMD protein level in mouse hippocampus CA1

426 region of control group, $\mathrm{SD}$ group, $\mathrm{SD}+\mathrm{SB} 203580$, and $\mathrm{SD}+\mathrm{U} 0126$ group $(\mathrm{n}=5)$ Results represent

427 the mean of three independent experiments $* p<0.05 . \mathrm{SD}$, sleep deprivation.

Peer] reviewing PDF | (2020:11:55599:2:0:NEW 17 May 2021) 


\section{Figure 1}

Figure 1. Sleep deprivation induced the increase of microglia and neuronal damage

Figure 1. Sleep deprivation induced the increase of microglia and neuronal

damage (A and B) IHC for IBA-1 level in hippocampus CA1 region of control group $(n=10)$, SD group $(n=10)$ and SD+ recover group $(n=10)$. Scale bars $=50 \mu \mathrm{m}$ for IHC. (C and D) H\&E staining for hippocampus CA1 region in control group $(n=10)$, SD group $(n=10)$ and SD+ recover group $(n=10)$. Scale bars $=100 \mu \mathrm{m}$ for $H \& E$. ( $E$ and F) Cell apoptosis were detected in hippocampus CA1 region in control group $(n=10)$, SD group $(n=10)$ and SD+ recover group $(n=10)$ by Tunel staining. Scale bars $=25 \mu \mathrm{m}$ for Tunel staining. Results represent the mean ofthreeindependentexperiments $* p<0.05$. IHC, immunohistochemical; IBA-1, Ionized calcium binding adaptor molecule-1; SD, sleep deprivation; H\&E staining, hematoxylin-eosin staining. 
A
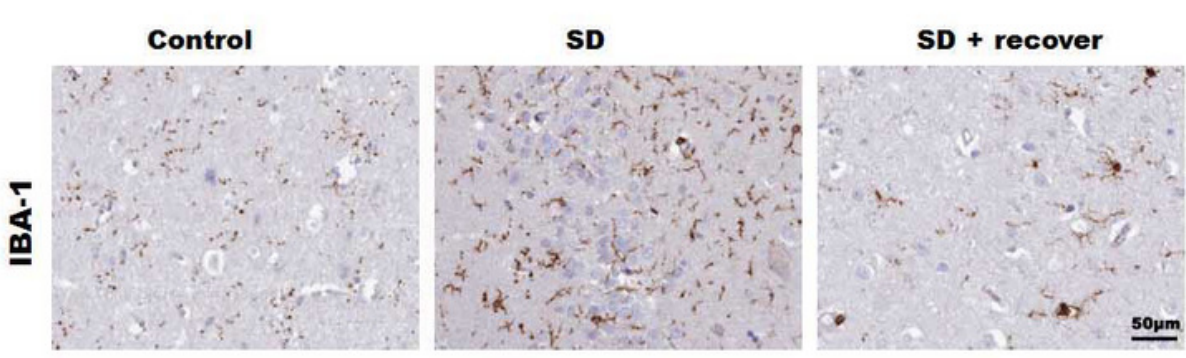

C
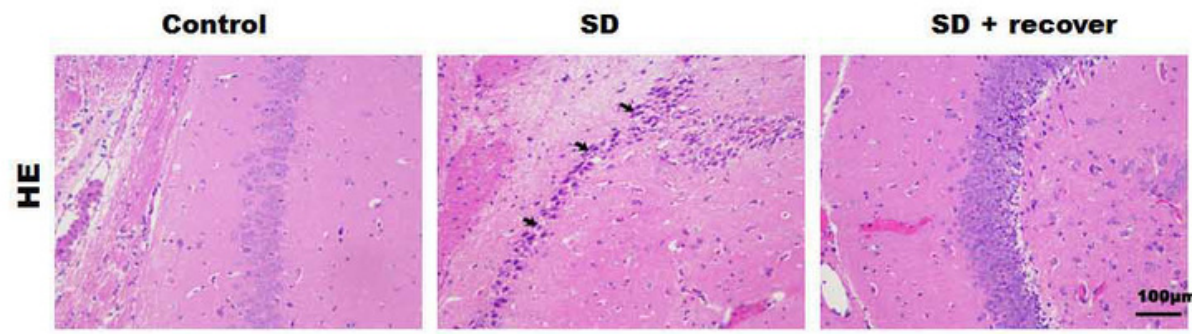

$\mathbf{E}$

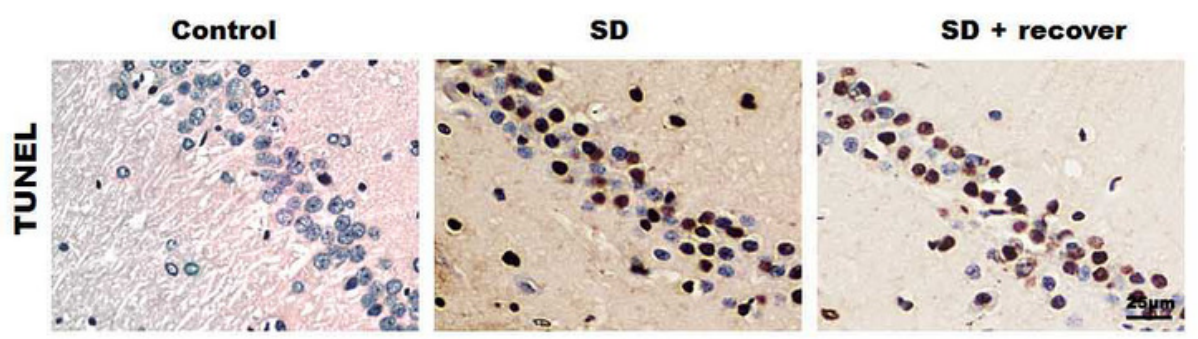

B

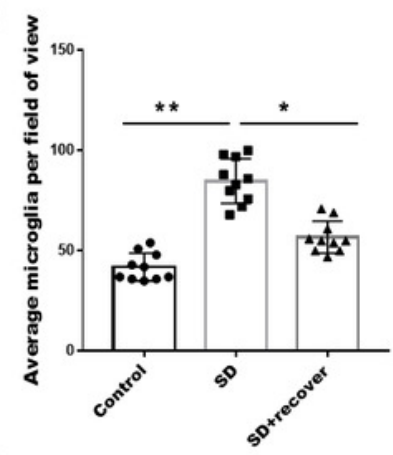

D

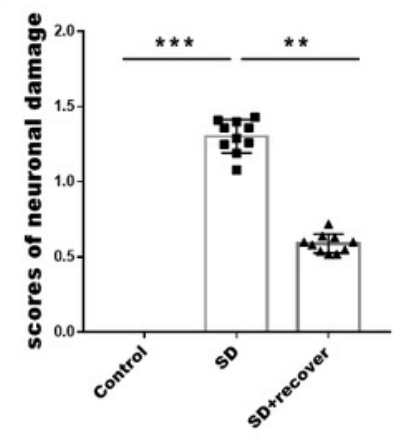

$\mathbf{F}$

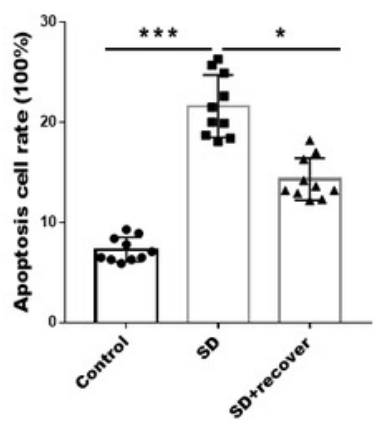




\section{Figure 2}

Figure 2. Sleep deprivation promoted the activation of NLRP3/caspase 1 pathway

Figure 2. Sleep deprivation promoted the activation of NLRP3/caspase 1

pathway(A) RT-PCR array for the expression levels of genes (IL-1 $\beta$, IL-18, Caspase-1, NLRP3, Caspase-4, ASC, GSDMD, NLRP1, NLRP2 and AIM2) in control group, SD group and SD+ recover group ( $n=5$ ). (B and C) Western blot analysis for Caspase-1, ASC and NLRP3 protein level in hippocampus CA1 region of control group, SD group and SD+ recover group $(n=5)$. (D) IHC for NLRP3 level in hippocampus CA1 region of control group, SD group and SD+ recover group. Scale bars $=50 \mu \mathrm{m}$ for $\mathrm{IHC}(n=5)$. Results represent the mean ofthreeindependentexperiments $* p<0.05, * * p<0.01$. RT-PCR, Quantitative real-time PCR; IHC, immunohistochemical; SD, sleep deprivation; ASC, apoptosis-associatedspecklikeprotein containing a CARD. 
A

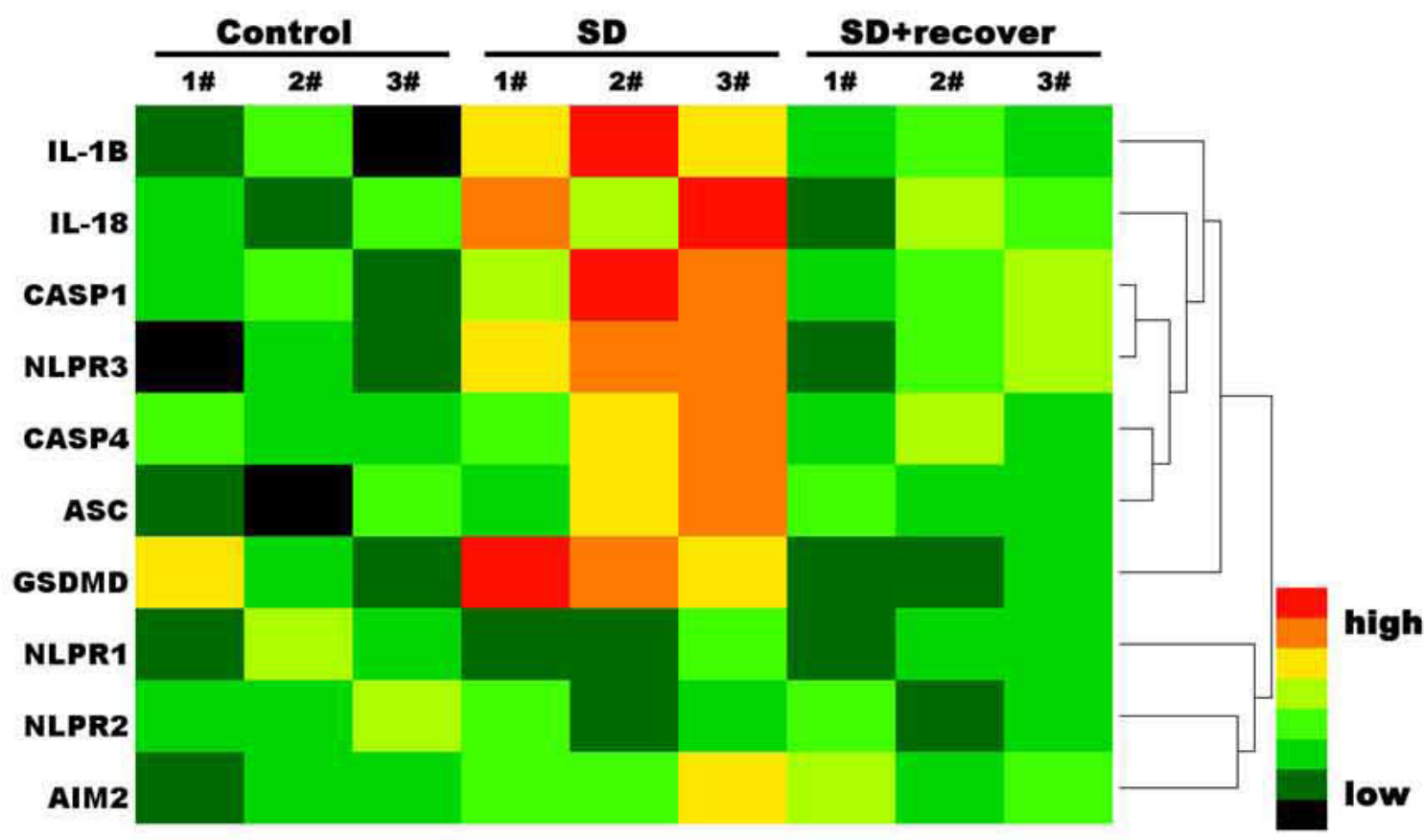

$\mathbf{B}$
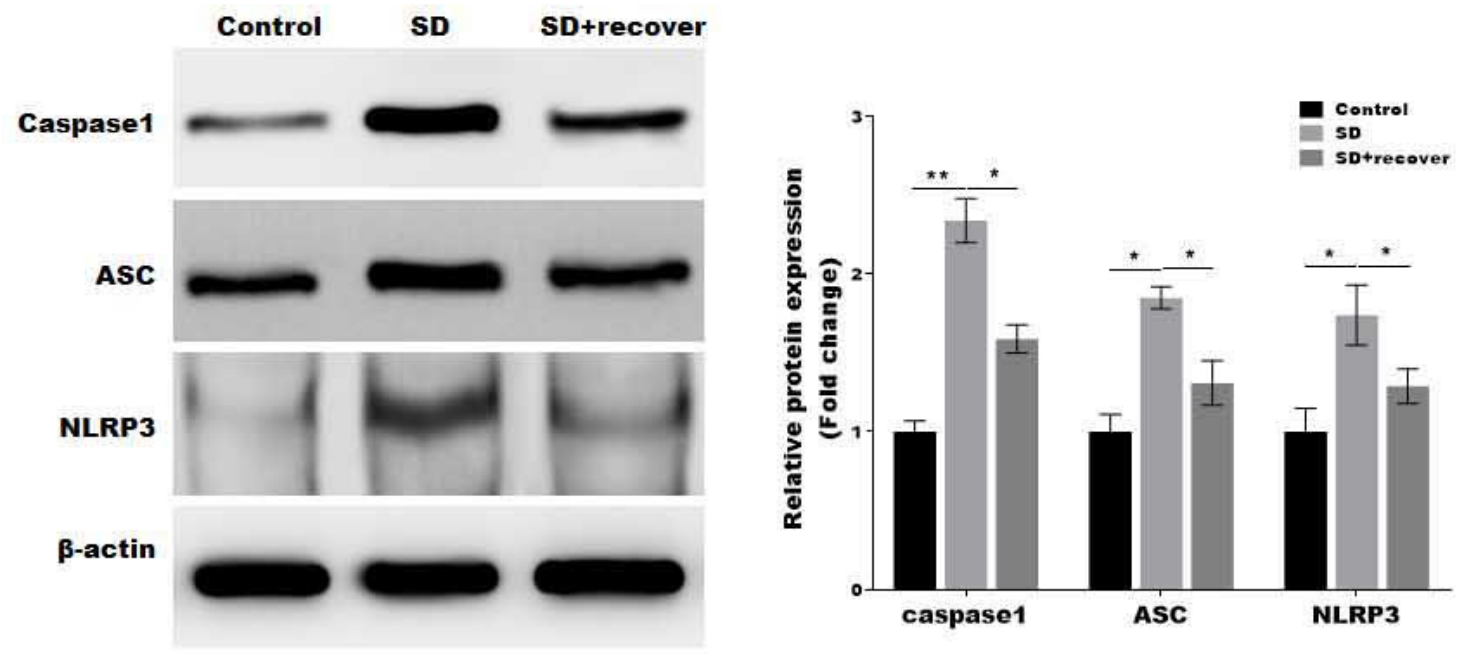

C

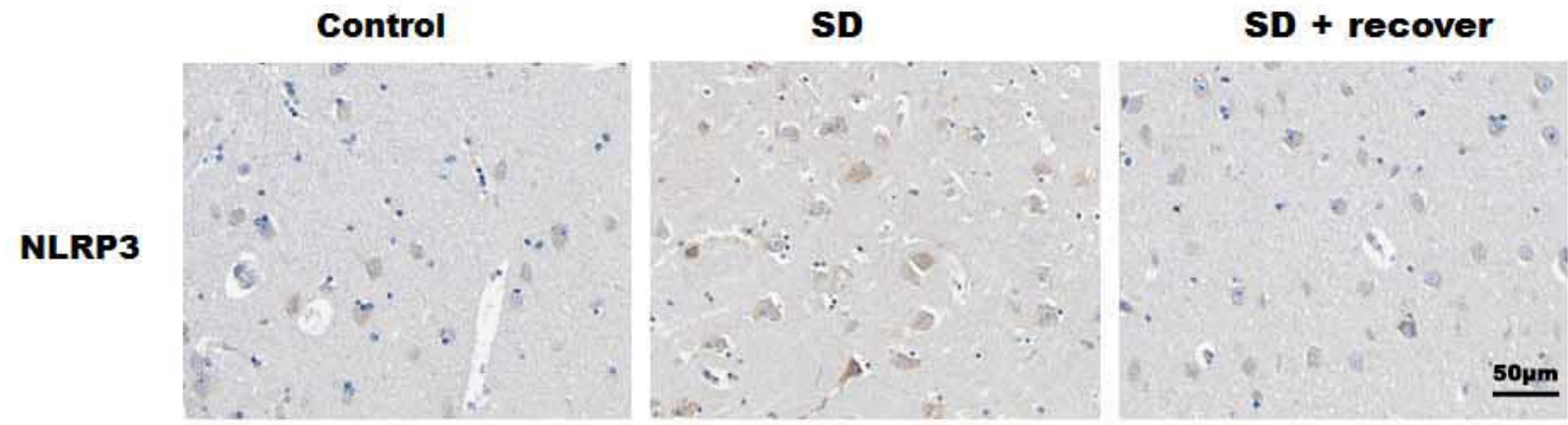




\section{Figure 3}

Figure 3. Sleep deprivation induced the pyroptosis of neuronal cell

Figure 3. Sleep deprivation induced the pyroptosis of neuronal cell(A and $B) q P C R$ (A) and ELISA (B) analysis of IL-1 $\beta$, IL-18 and TNF- $\alpha$ expression in hippocampus CA1 region of control group, SD group and SD+ recover group and qPCR analysis of IL-1 $\beta$, IL-18 and TNF- $\alpha$ expression in hippocampus of control group, SD group and SD+ recover group $(n=5)$. (C and D) Western blot analysis for GSDMD and bax protein level in mouse hippocampus CA1 region of control group, SD group and SD+ recover group $(n=5)$. (E) IHC for GSDMD level in mouse hippocampus CA1 region of control group, SD group and SD+ recover group $(n=5)$. Scale bars $=50 \mu \mathrm{m}$ for IHC. (F) Fluorescence co-localization for GSDMD and NeuN in mouse hippocampus CA1 region of control group, SD group and SD+ recover group $(n=5)$. Scale bars $=20 \mu \mathrm{m}$. Results represent the mean ofthreeindependentexperiments $* p<0.05$, ${ }^{* *} p<0.01$. qPCR, Quantitative real-time PCR; IHC, immunohistochemical; SD, sleep deprivation. 
A

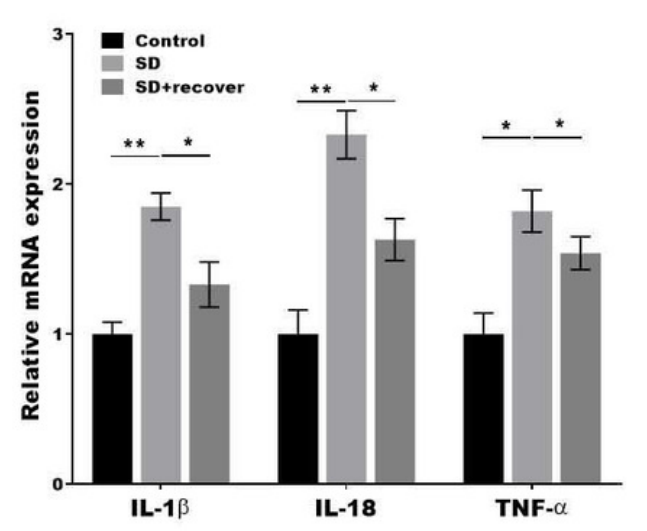

C

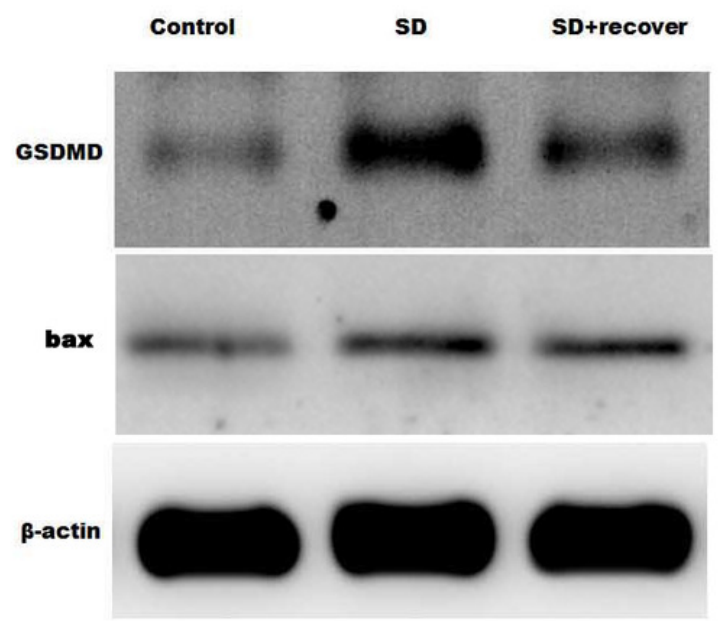

E
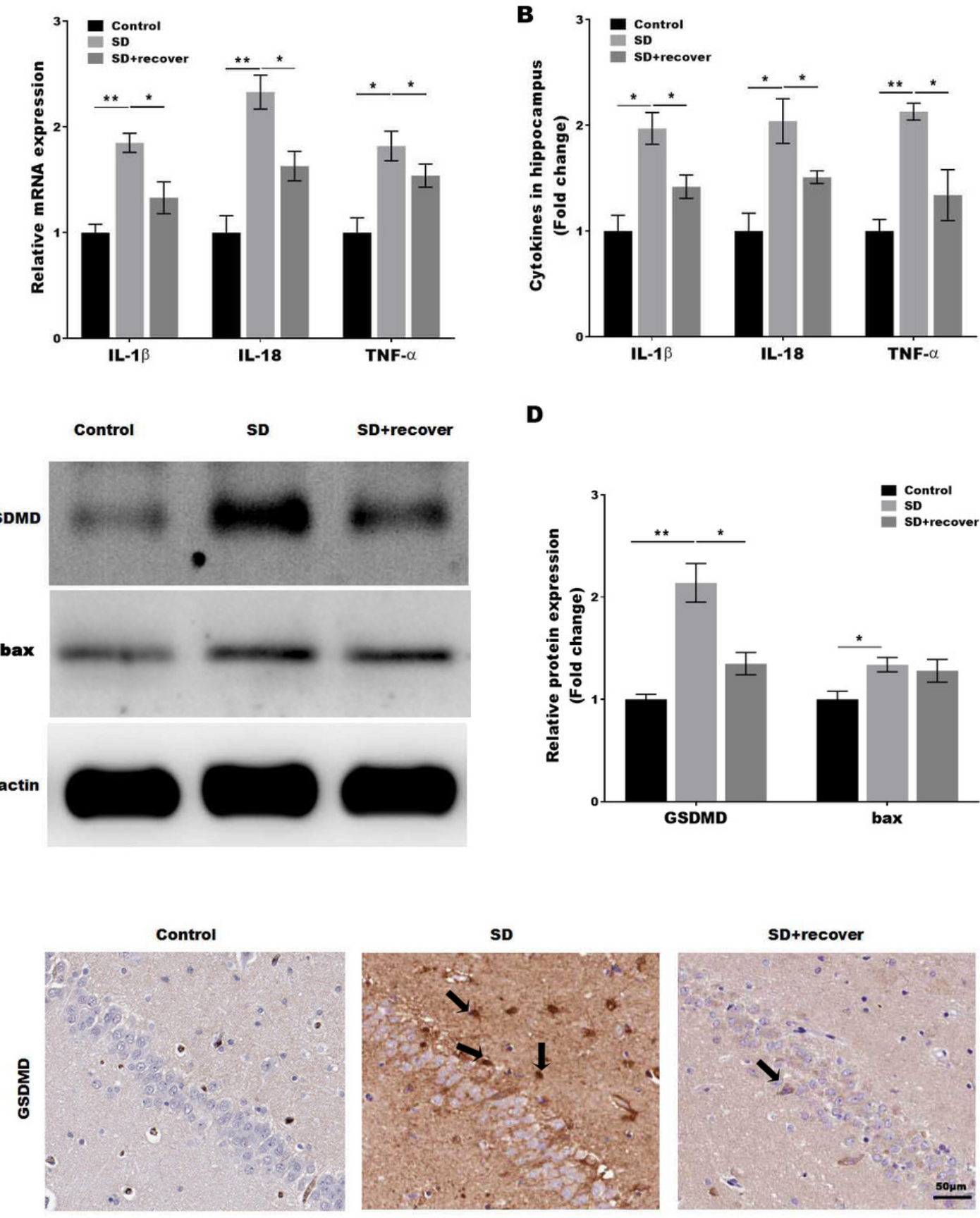

D

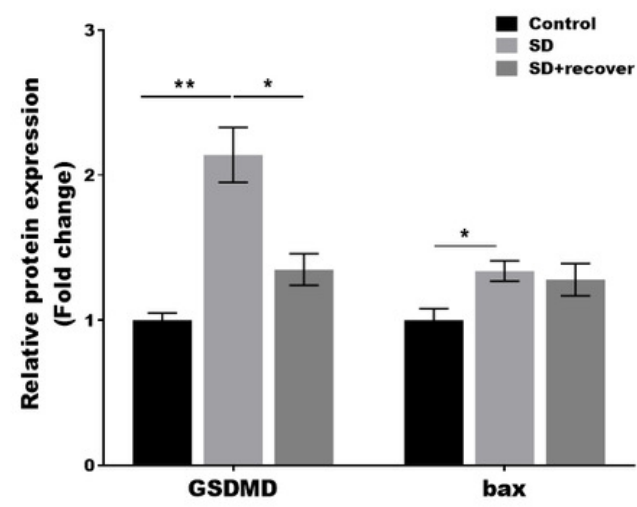

$\mathbf{F}$

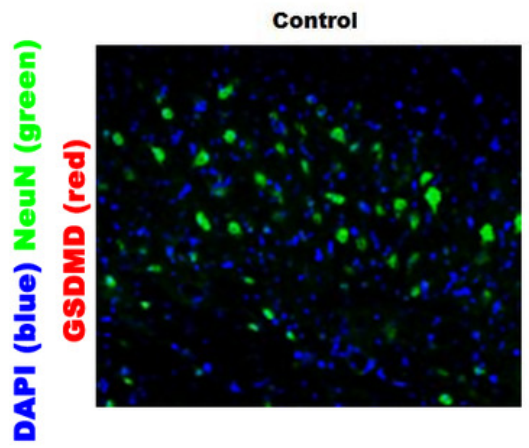

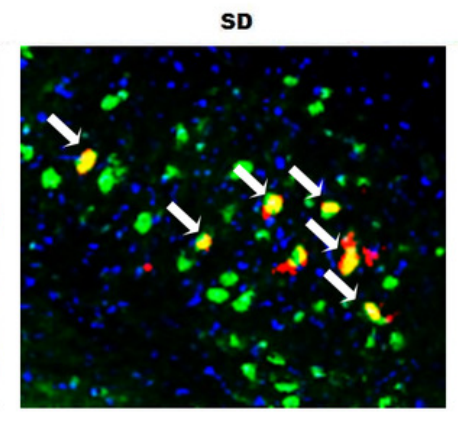

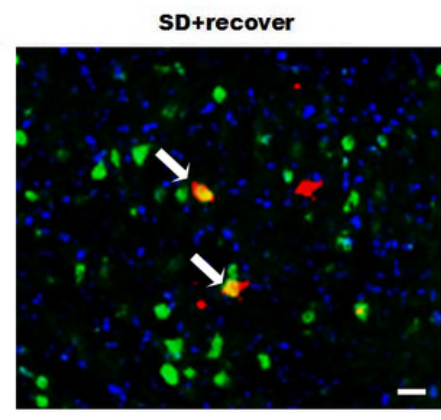




\section{Figure 4}

Figure 4 Sleep deprivation induces the activation of P38 and ERK MAPKs pathway

Figure 4. Sleep deprivation induces the activation of P38 and ERK MAPKs

pathway(A and B) Western blot analysis for p-P38 and P38 protein level in mouse

hippocampus CA1 region of control group, SD group and SD+ recover group $(n=5)$. (C and D) Western blot analysis for p-ERK and ERK protein level in mouse hippocampus CA1 region of control group, SD group and SD+ recover group $(n=5)$. (E and F) Western blot analysis for $p$ AKT and AKT protein level in mouse hippocampus CA1 region of control group, SD group and SD+ recover group $(n=5)$. ( $G$ and H) Western blot analysis for NLRP3 and GSDMD protein level in mouse hippocampus CA1 region of control group, SD group, SD+ SB203580, and SD+ U0126 group $(n=5)$ Results represent the mean ofthreeindependentexperiments $* p<0.05$. SD, sleep deprivation. 
A

C

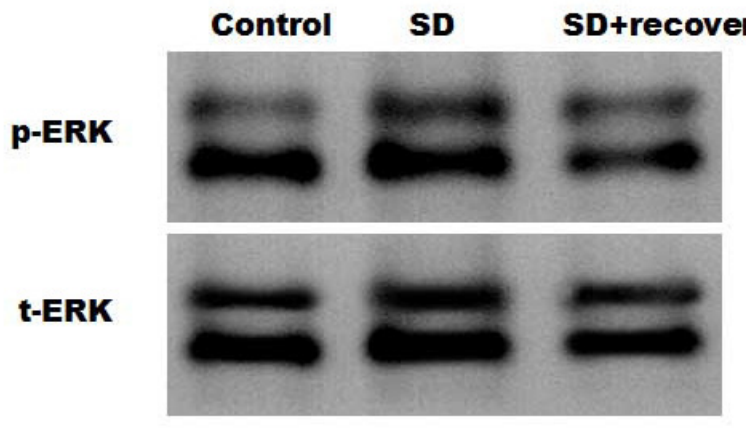

$\mathbf{E}$

t-P38
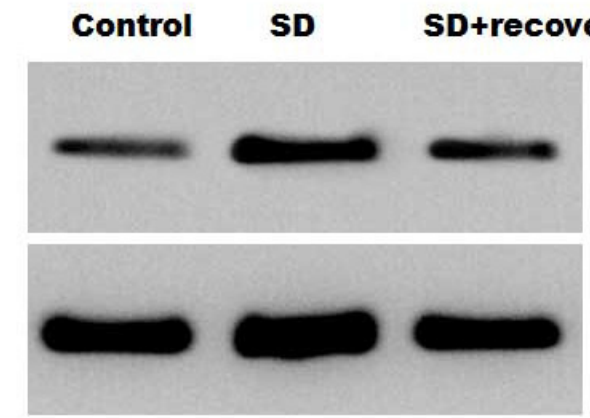

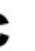

p-P38

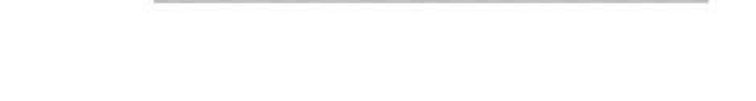

D
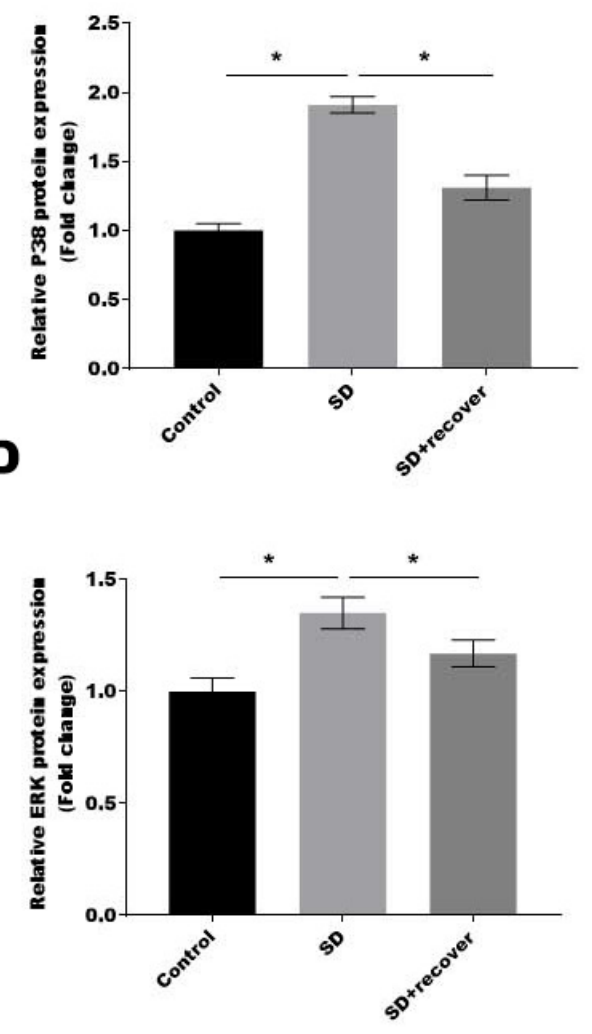

$\mathbf{F}$

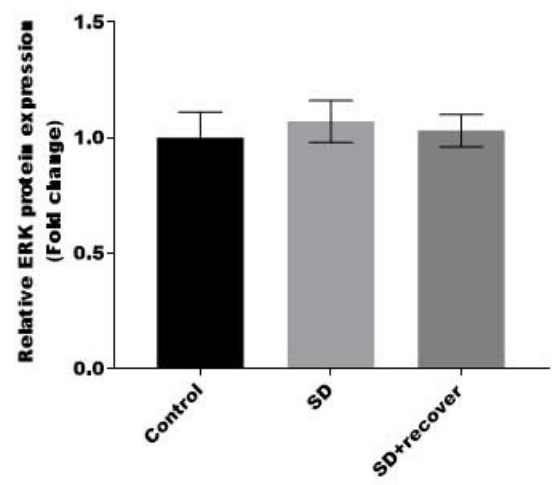

H

\section{Control SD SD+SB203580 SD+U0126}

NLRP3

GSDMD

B-actin

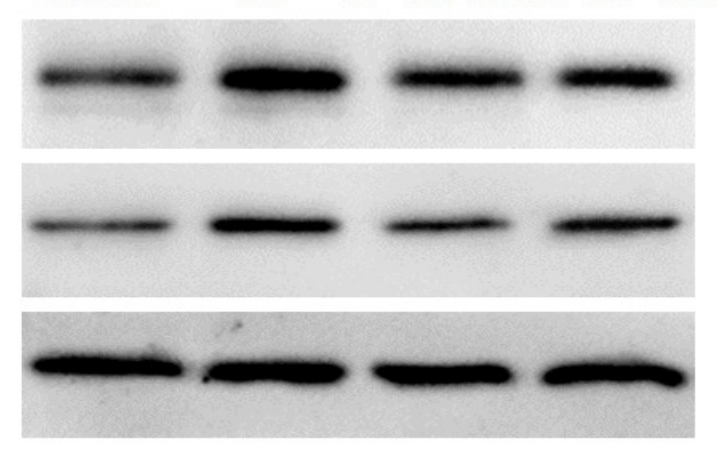

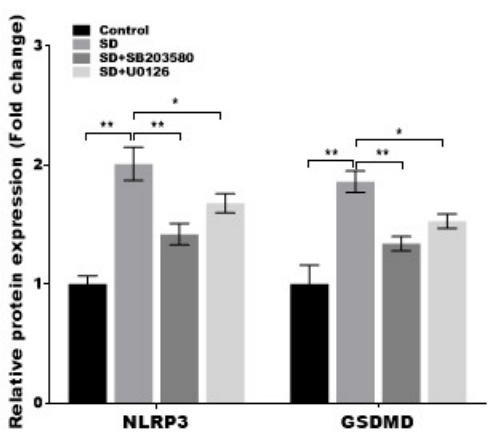

